\title{
EL DERECHO COMO PRÁCTICA Y CIENCIA SOCIAL: MÁS ALLÁ DEL SILOGISMO \\ Una réplica a Martín Rearte
}

\section{LAW AS PRACTICE AND SOCIAL SCIENCE: BEYOND SILOGISM A reply to Martin Rearte}

Mario A. Ramírez Granados*

\begin{abstract}
RESUMEN
El presente artículo contesta la concepción del derecho que propone Martín Rearte, mostrando los paradigmas internos del derecho, las prácticas sociales alrededor del derecho por el control de la interpretación legal y se propone un estudio de caso, a partir del funcionamiento de la Sala Constitucional Costarricense.
\end{abstract}

PALABRAS CLAVE: DERECHO * TEORÍA SOCIAL * SOCIOLOGÍA DEL DERECHO * IDEOLOGÍA $*$ PARADIGMA

\section{ABSTRACT}

This article replies Martin Rearte's definition of law. It shows the internal paradigm in the Law Science, its practices for the control of legal interpretation and proposes a studie case about the functions of Sala Constitucional de Costa Rica.

KEY WORDS: LAW $*$ SOCIAL THEORY * LAW SOCIOLOGY $*$ IDEOLOGIE $*$ PARADIGM 


\section{INTRODUCCIÓN}

Recientemente el profesor Martin Rearte introdujo en estas páginas la necesidad de discutir la relación del Derecho con otras ciencias, mediante el concepto de paradigma. En opinión de este autor sin embargo, el enfoque de Rearte no es completo, por lo que impide al lector entender el funcionamiento del Derecho como Ciencia, $y$ la forma en que se genera $y$ se transmite el conocimiento. Para ello se propone complementar este enfoque con el análisis de los paradigmas jurídicos internos.

En la primera parte del presente trabajo se pretende revisar la descripción que realiza Martin Rearte de las Revoluciones Científicas a la Ciencia Jurídica, problematizando la aplicación de esta teoría en la disciplina actual $y$ tratando de identificar algunos problemas propios de esta rama del conocimiento. En un segundo momento se proponen los paradigmas internos que funcionan al interior del Derecho, los cuales compiten por el control del sentido de la interpretación. En un tercer momento se procede a analizar el funcionamiento del derecho como práctica social. Finalmente en una cuarta unidad se busca ilustrar el debate entre los paradigmas jurídicos en las sentencias de la Sala Constitucional y mostrar someramente el estado actual de las Ciencias Jurídicas en América Latina.

\section{LA CONCEPCIÓN DEL DERECHO DE REARTE: LA CIENCIA JURÍDICA COMO CIENCIA FORMAL}

El artículo de Rearte analiza el cambio del concepto de ciencia en los diferentes paradigmas que han orientado el desarrollo del pensamiento racional. Por paradigma comprende "programas de investigación, lenguajes científicos, estructuras lingüísticas o marcos conceptuales" (Kuhn citado por Rearte, 2007: 198).

Se busca analizar la evolución del pensamiento a partir de la aplicación del concepto de paradigma en las ciencias naturales y sociales. El autor menciona parte de la concepción tradicional de ciencia, que asocia con el modelo de la Física newtoniana, donde "el mundo es concebi- do como un cosmos estable, ordenado por leyes físicas propias de las ciencias causales" (Rearte, 2007: 198). Dicho modelo tiene su equivalente en las formulaciones de la sociedad como orden en la filosofía política del momento (se enfatiza sobre todo en el caso de Hobbes).

Dicho modelo entra en crisis del surgimiento de nuevas corrientes al nivel de la Física (las ideas sobre la relatividad), la Lingüística (el surgimiento del concepto de juegos de lenguaje), superando la concepción clásica o positivista de la ciencia estrechamente ligada al concepto de orden, hacia el concepto de complejidad, donde se reemplaza el concepto de verdad universal por la idea de regularidad falseable, a partir de la aparición de nuevos hechos o teorías que permitan confirmar o refutarla.

A partir de la caracterización de los paradigmas, el autor critica al Derecho como una ciencia centrada en el método silogístico, indicando que la necesidad de dictar leyes causales $y$ universales no es extensible a las Ciencias Sociales, indicando "que ya no es posible el razonamiento silogístico en todos los casos y sin miramientos, dado que puede haber otras conclusiones o más de una" (Rearte, 2007: 203).

Sin embargo, en su argumentación, Rearte no da ejemplos que demuestren la imposibilidad del uso del silogismo en la construcción de la Ciencia Jurídica o el planteamiento de nuevos métodos de investigación que superen al silogismo.

Si bien Rearte indica algunos exponentes de lo que él denomina las corrientes integradoras del Derecho, como la Escuela Egológica de Carlos Cossio o el Tridimensionalismo de Miguel Reale. En su texto no se indica el grado de influencia sobre las Facultades de Derecho o los Institutos de Investigación Jurídica, que son las fuentes principales de investigación.

\section{LAS LIMITACIONES DE LA APLICACIÓN DE LOS PARADIGMAS A LA CIENCIA DEL DERECHO}

Al analizar la posibilidad de aplicar el modelo de paradigma a la Ciencia Jurídica que propone Rearte se considera que deben al menos responder a dos interrogantes: 
¿Cómo se aplican estos paradigmas en la investigación jurídica?

¿Cuáles son los paradigmas en el Derecho? ¿Y en qué forma actúan?

Respecto a la aplicación de estos paradigmas en la investigación jurídica, en el texto no se muestra claramente en qué forma estos cambios de paradigmas han influido en el desarrollo de la Ciencia Jurídica o las resistencias de esta a estos nuevos enfoques.

El primer problema en la aplicación del paradigma de las Ciencias Naturales a las Ciencias Jurídicas que realiza Rearte, es que estas no buscan descubrir objetos, sino que se trata de una ciencia que busca solucionar problemas de tipo valorativo. Esta situación se agrava porque el autor hace una forzosa generalización sobre el debate jurídico, dejando de lado las subdivisiones al interior del Derecho, así como su relación con ciencias cercanas, como la Sociología Jurídica, la Antropología Jurídica, entre otras.

En ese sentido, es importante indicar que la difícil relación entre el Derecho y las Ciencias ha sido tocada principalmente por la Filosofía del Derecho, pero en el texto se dejan de lado estas discusiones. Stammler indica que el Derecho no es un cuerpo físico ni es tampoco una fórmula conceptual para investigar los fenómenos del espacio, sino que se trata de aplicaciones y manifestaciones de la voluntad vinculativa de una sociedad (Sin fecha: 139).

Otro clásico de las Ciencias Jurídicas, Gustav Radbruch remite a que el Derecho no puede ser derivado inductiva y empíricamente de los fenómenos jurídicos, sino que se trata de un concepto cultural, que puede ser captado deductivamente (Cf: Radbruch, 2005: 46-47).

La obra en comentario no considera el debate durante los siglos XIX y xx, entre el Formalismo y el Antiformalismo la cual es una caracterización importante para entender la historia del pensamiento jurídico como Ciencia Social y la creación del campo a partir de la lucha entre corrientes que agotan el estudio del Derecho en la Ley misma o la crítica hacia la sobreutilización de este tipo de conceptos $y$ la reivindicación de factores extranormativos.
Dentro de estos ejemplos no hay referencias a Escuelas como la Jurisprudencia de Intereses, la jurisprudencia de conceptos o la Nueva Retórica (Perelman) o la polémica entre autores más recientes como Rawls, Alexy o Habermas.

En ese sentido si bien el autor indica el problema de la necesidad de enunciados generales en el Derecho, pero no aborda la problematización del grado de incertidumbre en la aplicación de las normas. Esta interpretación es definida por el llamado Realismo Norteamericano (Frank, Llewelyn) y en la actualidad por los llamados Critical Legal Studies.

Es decir, que en el texto no logra engarzar las discusiones sobre los diferentes paradigmas dentro del desarrollo de las Ciencias Jurídicas, ni se desarrollan los problemas propios del Derecho. Se deja de lado que en el Derecho existen discusiones abiertas, sobre conceptos o instituciones que se encuentran en disputa.

Las referencias antes mencionadas, no indican que el Derecho carezca de carácter científico sino que para poder captar la forma en que se produce, se actualiza y se transmite conocimiento, no puede ser captado tratando de ubicar al Derecho utilizando las fórmulas de otras Ciencias, sino que debe partirse del Derecho, entendiendo sus procesos de creación y acumulación del conocimiento y ponerlo en diálogo con los paradigmas científicos existentes.

Se obvia además que en el Derecho la interpretación del mismo, no sólo contiene hechos, sino que tiene un importante componente retórico, el cual Rearte deja de lado en su argumentación. En las siguientes líneas intentar abordar algunas de las consideraciones del autor, contraponiéndolas con un abordaje desde el Derecho como práctica social, tratando de mostrar las particularidades de este.

\section{EL DERECHO COMO PRÁCTICA SOCIAL}

La comprensión del Derecho como práctica social, nos obliga a tratar de identificar las relaciones que funcionan en su interior, los agentes que participan y los intereses 
que persiguen. En este sentido, siguiendo a Bourdieu, se parte del Derecho como un universo social relativamente independiente de las demandas externas (de otros campos sociales como la economía) al interior del cual se produce y se ejerce la autoridad jurídica (2002: 158).

Un primer aspecto es el funcionamiento cotidiano del Derecho. El intérprete jurídico no busca homologar el resultado de su producto como si hiciera Ciencia Jurídica, por el contrario, pretende primeramente solucionar una determinada situación, favoreciendo los intereses de la persona que representa. En ese nivel no busca hacer ciencia.

La elaboración científica es un proceso posterior, generalmente realizado por investigadores o profesores universitarios, mediante la sistematización de sentencias, la comparación de normas o instituciones de diferentes ordenamientos, tratando de generar conocimiento nuevo.

Al nivel de la práctica cotidiana, la práctica jurídica se caracteriza por la lucha entre al menos dos oponentes, por convencer a un tercero (sea el Juez, o un Tribunal) sobre la veracidad de la caracterización de los hechos en disputa en un caso determinado $y$ obtener un determinado resultado (en este caso la condena o la absolución de una persona acusada por un determinado delito; o que se obligue o no al pago de una indemnización por un daño provocado).

El conjunto de hechos objetivos, estados emocionales y políticas públicas que afectan los intereses jurídicos de una persona es lo que se denomina como caso (Cf. Díaz, 2006: 16-17). Este cuadro fáctico se va a contraponer con la presentación de hechos que expone la contra parte.

Partiendo desde una concepción jurídica, la norma es un juicio hipotético que consta de requisitos $y$ de efectos, que condicionan una cierta voluntad jurídica a un determinado hecho como su fundamento jurídico (Stammler, sin fecha: 396). Los componentes de dicha norma, pueden ser captados individualmente por la persona que interpreta, que trata de identificar cuál es el sentido de esta voluntad, cuáles son los distintos requisitos de los que se compone, a qué instituciones jurídicas remite.
Parte de estas respuestas pueden ser captadas directamente por la persona que interpreta, o en otros casos, como en los llamados conceptos jurídicos genuinos, es decir conceptos tomados del derecho positivo o conceptos puramente formales (Radbruch, 2005: 11-12). Este es el caso de términos como silencio administrativo, desviación de poder, familia, compraventa, los cuales preexisten a la norma que se aplica. Este tipo de análisis es lo que se denomina generalmente como análisis dogmático o dogmática jurídica.

Esta controversia se resuelve por medio de un tercero, en un nivel administrativo o judicial, al que se le denomina juez o mediador, que considera los argumentos de cada parte, analizando el grado de validez de su planteamiento $y$ dando una solución que pueda satisfacer totalmente a uno de los ponentes, o parcialmente ambos.

Es decir que en la práctica judicial, contrariamente a lo que indica Rearte, no existe una única solución basada en la comprobación de una determinada hipótesis, sino que se trata de la fuerza de argumentos que deben probar su validez, mediante las pruebas que presenta el intérprete. Igualmente en la práctica judicial no se aplica mecánicamente el silogismo, sino que primero se identifican qué hechos pueden probarse o no probarse, antes de que el juez construya el caso, a partir de las pruebas que presenten las partes en conflictos como documentos o testimonios que confirman su versión o que sirven para falsear los argumentos de la contraparte. Junto a los hechos, y las pruebas, se recurre a "criterios de política jurídica que le permitan solucionar aspectos no resueltos intersubjetivamente" (Véase Haba, 2005: 39).

En un segundo nivel, es importante indicar que dentro del Derecho como práctica social, existen procesos de control de resultados, mediante los tribunales superiores. Este control se refiere tanto a las conclusiones sobre los hechos, para lo cual se hace un control del análisis de las pruebas realizada por el Tribunal inferior, sino que también supone un control de los argumentos jurídicos.

Desde este punto de vista, los tribunales cumplen una doble función como instancia garante del derecho a la segunda instancia y 
encargado del control de la producción de las sentencias y su apego a la línea jurisprudencial.

A partir de la resolución de casos al nivel cotidiano, surge un tercer proceso, al que se denomina sistematización, actualización y enseñanza del derecho mediante la investigación y la docencia, las cuales realizan fundamentalmente las Universidades, puesto que estas instituciones son las que cuentan fundamentalmente con recursos como tiempo o bibliotecas.

La sistematización del derecho, consiste fundamentalmente en la identificación de reglas que puedan servir como parámetros para casos similares. Esta identificación de reglas se realiza mediante el análisis de las normas jurídicas, identificando y separando cada uno de sus componentes, mostrando las instituciones jurídicas presentes $y$ en algunas ocasiones analizando la figura jurídica presente en la norma con la dogmática jurídica o el derecho comparado. A partir de la identificación de estos tres niveles, al nivel de la práctica cotidiana, es que se puede analizar la lucha entre paradigmas jurídicos.

Dentro de este universo, las diferentes prácticas sociales que realizan los agentes que operen en dicho campo, comparten un rasgo en común: buscan que el interés o la posición que defienden o que portan se conviertan en la práctica jurídica legítima. La lucha entre formalistas y antiformalistas deja ver la lucha por "el control del monopolio de la interpretación legítima”, mediante el control de los principales medios para la actualización y sistematización del conocimiento jurídico, las Cortes y las Universidades. Esto implica que los intérpretes del derecho, sean abogados litigantes, profesores o jueces buscan que sus argumentos se conviertan en la ley, o la interpretación judicial correcta. Una segunda consecuencia es la posibilidad de desarrollar una arqueología de las instituciones u objetos jurídicos.

\section{ANÁLISIS DE CASO: LA LUCHA POR EL CONTROL DE LA INTERPRETACIÓN CONSTITUCIONAL EN EL CAMPO JURÍDICO COSTARRICENSE CONTEMPORÁNEO}

En las secciones anteriores se analizaron las propuestas del señor Rearte, quien plan- teó el problema del Derecho como una ciencia formal. Ante esta situación se indicó que el Derecho no se aplica en este momento como una reproducción mecánica del silogismo, sino que en la cotidianeidad el intérprete jurídico debe construir los hechos sobre los cuales se va a sustentar la posición que defiende. Esta construcción implica el uso de conceptos jurídicos, conceptos tomados de otras ciencias y criterios retóricos, para lograr conseguir el resultado y que la interpretación buscada sea considerada como legítima, lo que lleva a la lucha al interior del campo jurídico entre diferentes facciones por controlar el sentido "legítimo" de una institución jurídica.

Desde esta concepción hay que advertir que se han dado cambios importantes en la interpretación jurídica en muchos países latinoamericanos, a raíz de la creación y consolidación de los Tribunales Constitucionales. En el caso costarricense, a partir de la creación de la Sala Constitucional, como una sala autónoma del Poder Judicial encargada de la defensa de los derechos humanos mediante el Recurso de Amparo y de Habeas Corpus, así como el control de constitucionalidad de los actos de los poderes judiciales y el acceso gratuito de cualquier persona a esta sede; ha favorecido la judicialización de las diferentes facetas de la vida social, lo que ha provocado el estudio del derecho judicial y el interés por las limitaciones de la legislación para regular ciertas situaciones o sectores de la población.

Esta situación ha llevado a que el Tribunal Constitucional haya introducido nuevos contenidos a los artículos constitucionales, partir de la integración de Convenios Internacionales o de doctrinas extranjeras.

Este es el caso por ejemplo del debido proceso, doctrina derivada de los artículos 39 y 41 de la Constitución Política, para garantizar el control del proceso penal y que evoluciona hacia un parámetro de constitucionalidad de las leyes y de los actos del Poder Ejecutivo. La aplicación del debido proceso, ha implicado el desarrollo judicial del Derecho Parlamentario, eliminando normas especiales que se introducían dentro de la Legislación ordinaria (a partir del desarrollo del principio de conexidad entre 
el proyecto legislativo presentado y la norma aprobada), desarrollando las potestades del legislador (mediante la delimitación del derecho de enmienda a los proyectos legislativos).

Otro ejemplo es la derivación judicial de los derechos a los servicios públicos, a la salud, $y$ el acceso a los medicamentos, como contenidos del Derecho a la Vida, a pesar de que en el texto constitucional no existe una referencia expresa a dichas cláusulas.

Este tipo de actuaciones por parte del Tribunal Constitucional ha llevado a que se acuse a dicho órgano de activismo judicial. La consecuencia de esta situación es la comprensión de los derechos constitucionales como instituciones en construcción, que se encuentran en disputa entre diferentes sectores de la comunidad, que tratan de incorporar sus puntos de vista dentro de la ley vigente, sea apegándose al sentido del texto, sea derivando nuevos contenidos a partir de la actualización de la Constitución. En ese sentido, los resultados de esta lucha se dan a partir de que una vez incorporados dentro del sistema jurídico, estos grupos pueden formular sus demandas como reclamos justificados en la razón.

En ese sentido se indica que:

Los derechos constitucionales están a caballo. Son tanto derechos legales insertos en y moldeados por la práctica argumentativa jurídica (las reglas legales) como entidades que "existen antes que $y$ fuera de la Constitución”. Por esta razón un argumento de derecho constitucional media no sólo entre juicios fácticos $y$ valorativos, sino también entre argumentos jurídicos (bajo un deber de fidelidad interpretativa) y argumentos legislativos (una apelación a los valores políticos de la comunidad) (Kennedy, 2006: 55).

Dentro de esta tendencia actual, se problematiza el concepto clásico de la interpretación judicial, enfatizando las lagunas, conflictos y ambigüedades del ordenamiento jurídico, y se centra en el papel del juez como ente encargado de resolver cotidianamente problemas jurídicos, dando un nuevo sentido, o completando el sentido establecido por el
Legislador. A esta interpretación se le conoce como la teoría de la Constitución viviente (Sagüés, 2006: 23).

Al nivel latinoamericano dentro de los representantes de esta tendencia se encuentran: César Rodríguez, Diego López Medina, Rodrigo Uprimmy, en Colombia y Néstor Pedro Sagüés, Cristian Curtis y Roberto Gargarella en la Argentina; entre otros.

Estos autores comparten la visibilización del rol político del derecho, cuestionando su supuesta neutralidad y mostrando el papel activo del intérprete, mostrando como se pueden dar a los textos jurídicos, interpretaciones distintas a su contexto de origen, dependiendo de los materiales jurídicos y de los criterios de conveniencia política (Kennedy: 2002).

Algunos de los problemas que laboran se centran en el estudio crítico de la enseñanza del Derecho y las tendencias jurisprudenciales (López Medina); el activismo judicial y la judicialización de la política (Uprimmy), la pluralidad jurídica y la ineficacia y el autoritarismo jurídico (Rodríguez).

Esta somera caracterización pretende mostrar que comprender al Derecho como un campo, implica romper con la idea del derecho como ciencia formal y mostrar que cotidianamente existen fuerzas en pugna que compiten por el Monopolio de la interpretación jurídica. Implica que se debe romper con la concepción del derecho como ciencia ahistórica, la interpretación jurídica se encuentra condicionada por la historia personal del intérprete, la tradición cultural de la que proviene y los intereses que porta $y$ defiende. En otras palabras, implica comprender que el debate sobre el Derecho es inacabado, actual y que puede enriquecerse con el aporte de otras disciplinas de las Ciencias Sociales.

\section{CONCLUSIONES}

En efecto el Derecho es una Ciencia Social, por su formación social y su objeto, históricamente utilizó al silogismo. Pero esto no significa reducir al Derecho a una aplicación mecánica del silogismo, o que se pretenda asimilarlo con los paradigmas científicos existentes, puesto que no puede captar la complejidad 
de relaciones que existen al interior del fenómeno jurídico.

Como se ha intentado mostrar, el Derecho es una ciencia valorativa, diseñada principalmente para la resolución de problemas, la cual puede ser captada desde su funcionamiento como práctica social. Desde la práctica social jurídica se identifican los diferentes actores $y$ niveles en los que funciona el campo jurídico, dichos actores convergen en la lucha para que su punto de vista se convierta en el punto de vista legítimo y se imponga al resto de los miembros de esa sociedad.

Desde ese sentido, entender la historia de una institución jurídica, implica también problematizar si los objetivos sociales en los que se basan estas normas se cumplen, cuál es su contexto ideológico de procedencia e identificar los cambios en ese discurso, tratando de visibilizar los grupos oprimidos o desaventajados. $\mathrm{O}$ siguiendo a Bourdieu (2002), que incorpora dentro de la enseñanza del Derecho, la deconstrucción del sentido universal de las normas, identificando relaciones ocultas que se encuentran en la estructura de las normas jurídicas.

\section{BIBLIOGRAFÍA}

\section{ARTÍCULOS DE REVISTA}

Haba, Enrique Pedro. “¿Puede el jurista discurrir como un científico social?". Revista de Ciencias Sociales 113-114. Universidad de Costa Rica, 2005: 37-54.

Kennedy, Duncan. "La crítica de los Derechos en los Critical Legal Studies". Revista Jurídica 47. Universidad de Palermo, 2006: 27-89.
Rearte, Martín. "El cambio de paradigma científico y el derecho". Revista de Ciencias Sociales 117-118. Universidad de Costa Rica, 2007: 197-210.

LIBROS

Bourdieu, Pierre. La fuerza del Derecho. Primera reimpresión. Santafé de Bogotá: Ediciones Uniandes. Siglo del Hombre Editores, 2002.

Díaz, Eduardo. Resolver casos, quid de la labor forense del abogado. Díaz, Eduardo (director): Como resolver casos. Esencia de la abogacía. Buenos Aires: Editorial Hammurabi, 2006.

Radbuch, Gustav. Introducción a la filosofía del Derecho. Novena reimpresión. México: Fondo de Cultura económica, 2005.

Rodríguez, César. Una crítica contra los dogmas de coherencia del derecho $y$ neutralidad de los jueces. En: Kennedy, Duncan: Libertad y restricción en la decisión judicial. Santafé de Bogotá: Ediciones Uniandes. Siglo del Hombre Editores, 2002.

Sagüés, Nestor Pedro. La interpretación judicial de la Constitución. Segunda Edición. Buenos Aires: Lexis Nexis-Abeledo Perrot, 2006.

Stammler, Rudolf. Tratado de Filosofía del Derecho. Madrid, Editorial REus: (Sin fecha). 